\title{
Evaluation of a Water Channel-Based Platform for Characterizing Aerostat Flight Dynamics: A Case Study on a Lighter-Than-Air Wind Energy System
}

\author{
Chris Vermillion* \\ University of North Carolina at Charlotte, Charlotte, NC 28223, United States, \\ Altaeros Energies, Somerville, MA 02143, United States, \\ Ben Glass ${ }^{\dagger}$ \\ Altaeros Energies, Somerville, MA 02143, United States, and \\ Sam Greenwood ${ }^{\ddagger}$ \\ University of Michigan Department of Aerospace Engineering, Ann Arbor, MI 48109, United States
}

\begin{abstract}
Aerostat development and testing costs often suffer from a lack of scalability. In particular, it very difficult to fabricate an inexpensive lighter-than-air system that can be evaluated in a lab environment, since the maximum allowable mass of the aerostat becomes prohibitively low for small length scales. This paper presents an evaluation of a novel water channel-based platform for assessing the flight dynamics of aerostats at a very small scale, in a lab environment, for a very low cost. Altaeros Energies' buoyant airborne turbine (BAT) is used as a case study to demonstrate the effectiveness of the proposed approach. Specifically, we identify important dynamic scaling properties and show how the water channel experiments are run to match these properties closely in the water channel vs. full-scale settings. We then show how the water channel results can be used in concert with a simulation model to predict the performance of the full-scale system. The ultimate result is a design which, after an inexpensive evaluation process, can proceed to a larger-scale prototype stage with a high degree of confidence in its success.
\end{abstract}

\section{Nomenclature}

$R_{A / B} \quad$ Aerodynamic to buoyant force ratio

$\lambda \quad i^{\text {th }}$ eigenvalue of the linearized system

Re Reynolds number

$v_{\text {flow }} \quad$ Flow velocity (of air or water)

$C_{\text {aero }} \quad$ Generic aerodynamic force coefficient

$A_{\text {ref }} \quad$ Reference area

$V_{\text {aerostat }}$ Aerostat volume

$s_{l} \quad$ Model length scale factor

$s_{l t} \quad$ Tether length scale factor

$l_{\text {chord }} \quad$ Chord length

$\rho \quad$ Fluid density

$\mu \quad$ Dynamic viscosity

$\nu \quad$ Kinematic viscosity

\footnotetext{
*Assistant Professor and Technical Advisor

${ }^{\dagger}$ Chief Technical Officer

${ }^{\ddagger}$ Undergraduate Research Assistant
} 


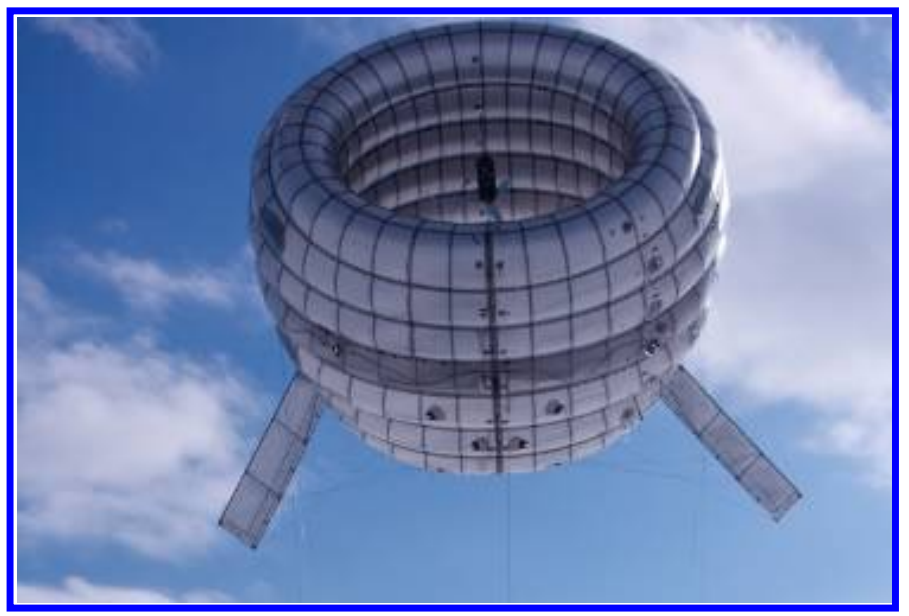

Figure 1. Altaeros Energies' first Buoyant Airborne Turbine (BAT) prototype in flight.

\section{Introduction}

Because aerostat volume scales with the cube of its length scale and the material surface area requirement scales with the square, it is challenging, time consuming, and expensive to build aerostats on a small scale, particularly when the goal is to build a prototype that can fly in an indoor lab environment. And while it is possible to build lab-scale demonstrations that fly in an inverted (upside-down) configuration, doing so makes the system negatively buoyant and reduces the contribution of added mass effects that are known to be important to aerostat flight dynamics. ${ }^{1-9}$ Consequently, aerostat designers often rely heavily or exclusively on computational fluid dynamics (CFD), dynamic simulation models, and lessons learned from past aerostat designs. Indoor, lab-scale demonstrations have largely been limited to systems that possess a standard geometry that requires little added structural shape to achieve (an elliptical aerostat or balloon) and still require significant resources and lab space. ${ }^{10}$

Fortunately for many aerostat designers, the traditional teardrop-shaped aerostat has been studied throughout the past century, and extensive results have been reported in the areas of flight dynamics ${ }^{1-9}$ and rules of thumb for aerostat design..$^{3,8}$ The dynamic models developed in the past century provide a framework that typically enables sufficiently accurate simulation and analysis of aerostat flight dynamics, given that aerodynamic, inertial, and added mass parameters are well-understood, and provided that simulation results are corroborated with years of design rules of thumb.

For small adaptations of the classic teardrop-shaped design, the inputs to standard aerostat models can indeed be quantified with satisfactory accuracy, and simulated behavior can be corroborated with years of actual aerostat deployment. Recently, however, several aerostat designs have emerged that mark a significant departure from the classic teardrop design. Some of these designs, such as Global Near Space System's Star Tower, ${ }^{11}$ are designed to achieve greater aerodynamic lift to achieve higher altitude operation at a limited zenith ("blowdown") angle. Others, such as Altaeros Energies' Buoyant Airborne Turbine (BAT), ${ }^{12}$ shown in Fig. 1, serve an unconventional purpose such as the generation of energy at high altitudes, and must assume a unique shape in order to achieve this purpose. In these cases, properties such as the added mass matrix and unsteady flow effects are difficult to quantify, and few rules of thumb exist from which a robust design can emerge. For these systems, it is desirable to iterate quickly and cheaply through a series of designs in an accurate experimental framework that can also be used to refine simulation models. Unfortunately, because aerostat lifting volume scales with the cube of length scale and material surface area (and, roughly speaking, weight) scales with length scale squared, it is very difficult to scale aerostats to lab-scale, particularly when those aerostats assume non-elliptical shapes (such as the BAT or GNSS Star Tower) that require substantial structural elements to maintain.

This paper examines a water channel-based framework that is used to rapidly and inexpensively evaluate aerostat flight dynamics in a lab-scale environment. As a case study, we show how the water channel-based framework has been applied to the Altaeros Energies BAT, in which an annular aerostat is used to elevate a horizontal axis turbine to high altitudes where wind speeds far exceed those at ground level. The system's 


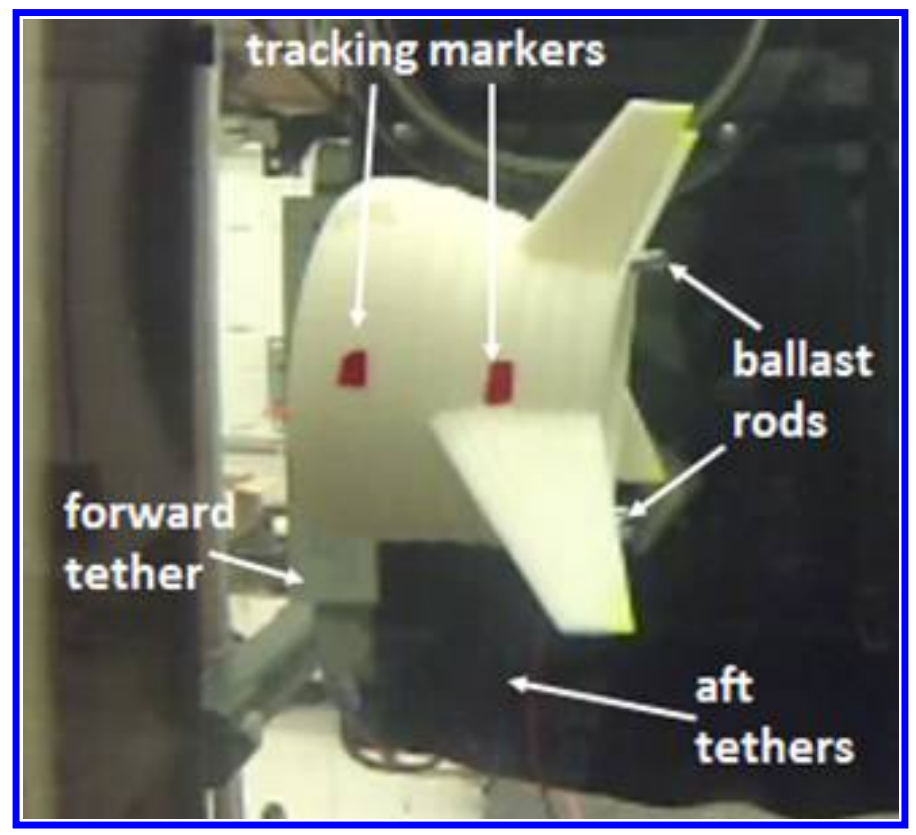

Figure 2. Photograph of the water channel experimental setup.

unique shape, coupled with the presence of a spinning rotor, which acts as a large drag source, lead to unique design and analysis challenges that are not encountered with conventional aerostats and are difficult to tackle in simulation alone. We begin in Section II with a description of the experimental hardware setup, including the procedure for designing flexible models that can be ballasted to achieve a variety of centers of mass and inertial properties. In Section III, we identify important dynamic scaling properties, specifically:

- Reynolds number;

- aerodynamic-to-buoyant force ratio;

- eigenvalues.

We show how these scaling properties compare in the water channel and full-scale settings. In Section IV, we show how the water channel results can be used in concert with a simulation model to predict the performance of the full-scale system, and how the water channel results can be used to refine the dynamic model.

\section{Experimental Setup}

For its testing, Altaeros Energies partnered with the University of Michigan, whose Aerospace Engineering department has a $2 \mathrm{ft} \times 2 \mathrm{ft}$ water channel capable of flow speeds up to approximately $0.5 \frac{\mathrm{m}}{\mathrm{s}}$. Within this water channel, 0.0075-scale models were tethered to a "ground station" at the bottom of the channel and routed up to translation stages above the water that could be used to adjust the aerostat trim, along with load cells to measure tether tensions during flight. Motion was captured using iOn AirPro side-view and top-view waterproof cameras. Fig. 2 shows the configuration in the water, during a test run, and Fig. 3 shows the frame (including the ground station and translation stages) that the models were tethered to.

To produce representative flight behavior in the channel, 0.0075-scale models were $3 \mathrm{~d}$ printed out of ABS plastic, using a low-density fill that yielded specific gravities of approximately 0.5. Each model contained ballasting holes, which could either remain empty or be filled with lead rod. This enabled variation of the center of mass and moments of inertia in order to characterize the sensitivity of dynamic performance to changes in inertial properties. Tests were conducted with and without a mesh drag screen inserted in order to understand the approximate effect of the rotor. 


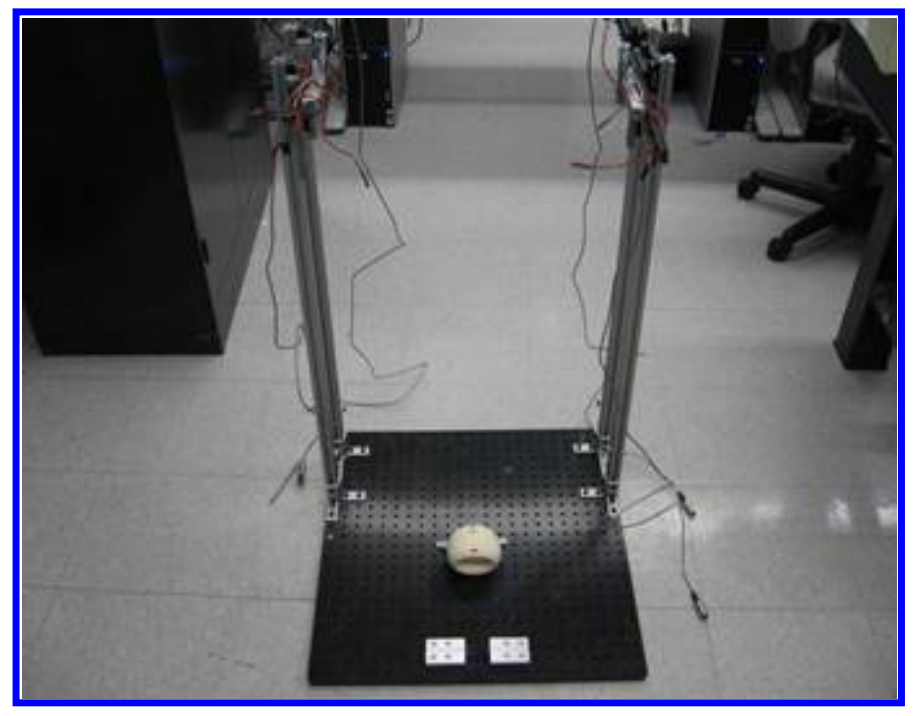

Figure 3. Photograph of the supporting test frame, including the ground station, translation stages, and load cells, out of the water.

\section{Dynamic Scaling}

When we scale a system by a factor as small as 0.0075 and move from air to water, it is essential that we understand how important system properties change with scaling and fluid. In particular, there are three properties that are of significant interest:

1. Aerodynamic-to-buoyant force ratio $\left(R_{A / B}\right)$

2. Linearized system eigenvalues $\left(\lambda_{i}\right)$

3. Reynolds number $(R e)$ - This familiar dimensionless ratio characterizes the dominance of either inertial or viscous effects in the flow and can be used to characterize the boundary layer as laminar or turbulent.

With all of these properties, we are interested in how the properties scale with changes in the model length scale, $s_{l}$, the fluid speed, $v_{\text {flow }}$, and the fluid properties, including density $(\rho)$ and viscosity $(\mu)$.

\section{A. Aerodynamic-to-Buoyant Force and Moment Ratios}

All aerostats, including the Altaeros BAT, are subjected to both aerodynamic and buoyant forces, which can have complementary or conflicting effects. At low wind speeds, buoyant forces typically dominate, and at high speeds, aerodynamic forces typically dominate, but the threshold at which wind speeds become "high" depends on the geometry and scaling.

Aerodynamic forces are given by:

$$
F_{\text {aero }}=\frac{1}{2} \rho v_{\text {flow }}^{2} C_{\text {aero }} A_{\text {ref }} .
$$

Because $A_{r e f}$ is proportional to $s_{1}^{2}$, it is immediately clear that:

$$
F_{\text {aero }}=k_{1} \rho v_{\text {flow }}^{2} s_{l}^{2},
$$

where $k_{1}$ is a constant that is independent of the fluid properties, length, or flow speed scaling.

Buoyant forces are given by:

$$
F_{\text {buoyant }}=\rho V_{\text {aerostat }}=k_{2} \rho s_{l}^{3} .
$$

From (2) and (3), we can immediately see that the aerodynamic-to-buoyant force ratio is given by:

$$
R_{A / B}=k_{3} \frac{v_{\text {flow }}^{2}}{s_{l}}
$$


where $k_{3}=\frac{k_{1}}{k_{2}}$. Thus, if one wishes to replicate aerodynamic-to-buoyant force ratios on a scaled model, he/she should run the water channel at a flow speed that is a factor of $\sqrt{s_{l}}$ times the target full-scale aerostat wind speed. It can easily be verified that the aerodynamic-to-buoyant moment ratio scales in the same way as the force ratio.

\section{B. Linearized System Eigenvalues}

Because an aerostat possesses 6 degrees of freedom in passive flight when tethers are treated as compliant, and $6-\min (n, 3)$ degrees of freedom when tethers are treated as kinematic constraints $(n$ is the number of tethers), its dynamics cannot be characterized by a single natural frequency or time constant, but instead must be characterized by multiple frequencies. To gain an understanding of how time constants vary with scaling properties, we examine the eigenvalues of the linearized system, where the linearization is performed about a downwind condition. For reference, we consider the Altaeros BAT, under different length and velocity scalings, where the treatment of tethers as kinematic constraints leaves 3 remaining degrees of freedom and 6 eigenvalues. Two of these eigenvalues correspond to translational longitudinal motion (the "pendulum" mode), two correspond to translational lateral motion (the "sway" mode), and two correspond to rotational motion about the tether axis, with complete decoupling between longitudinal and other modes.

While the precise scaling of eigenvalues with model length scale, tether length scale, and flow speed represents a complicated derivation that is beyond the scope of this work, some very robust general trends can be observed by examining eigenvalues at different linearization points. In particular, at high flow speeds, where aerodynamic forces dominate, we have found that the eigenvalues correlating most strongly with translational motion scale as:

$$
\lambda_{i} \approx k_{4} \sqrt{\frac{v_{\text {flow }}^{2}}{s_{l} s_{l t}}},
$$

where $k_{4}$ is independent of the fluid characteristics, length scale, and flow speed scale. The eigenvalues correlating most strongly with rotational motion scale similarly in this aerodynamic regime, as:

$$
\lambda_{i} \approx k_{5} \sqrt{\frac{v_{\text {flow }}^{2}}{s_{l}^{2}}} .
$$

At lower flow speeds, where buoyant forces dominate, we have found that the eigenvalues correlating most strongly with translational motion scale as:

$$
\lambda_{i} \approx k_{6} \sqrt{\frac{1}{s_{l t}}}
$$

The eigenvalues correlating most strongly with rotational motion scale similarly in this buoyant regime, as:

$$
\lambda_{i} \approx k_{7} \sqrt{\frac{1}{s_{l}}} .
$$

Thus, in high flow speeds, if one wishes to replicate time constants on a scaled model, he/she should run the water channel at a flow speed that is a factor of $\sqrt{s_{l} s_{l t}}$ times the target full-scale aerostat wind speed to replicate translational time constants but should run the water channel at $s_{l}$ times the target full-scale speed to replicate rotational time constants. At lower flow speeds, where buoyant forces dominate, one will always observe faster time constants for smaller length scales and cannot use the water channel speed as a compensation mechanism. Table 1 gives a comparison of system eigenvalues for a particular ballast configuration of the aforementioned full-scale Altaeros BAT model and a scaled-down version to match water channel scaling of $s_{l}=0.0075, s_{l t}=0.0022$ and corresponding fluid properties. From this table, one can see that eigenvalues of the scaled model generally fall within or near the expected values, according to the scaling relationships of (5)-(8).

We have established a connection between eigenvalues of the full-scale and water channel-scale system, and have shown that the trends remain the same whether one considers translational or rotational modes. Nevertheless, the quantitative scaling factors are different for translational and rotational motion, and scaling factors depend on whether the dynamics are dominated by buoyant or aerodynamic forces. Clearly, some sacrifices must be made, and small-scale behavior can never be made to perfectly match full-scale behavior. Section IV discusses how these sacrifices have been handled on the Altaeros BAT. 
Table 1. Comparison of eigenvalues at full-scale and water channel-scale, with a comparison between calculated eigenvalues and those approximated by (5)-(8).

\begin{tabular}{rrrrr} 
& $\begin{array}{r}\text { Full-scale } \\
\text { scale } \lambda_{i}\end{array}$ & $\begin{array}{r}\text { Water channel- } \\
\text { channel } \lambda_{i}\end{array}$ & $\begin{array}{r}\text { Expected water channel } \lambda_{i} \\
\text { - aerodynamics-dominant }\end{array}$ & $\begin{array}{r}\text { Expected water channel } \lambda_{i} \\
\text { - buoyancy-dominant }\end{array}$ \\
\hline Longitudinal & -0.199 & -4.278 & -2.18 & -4.20 \\
(pendulum) & -0.395 & -7.144 & -4.33 & -8.33 \\
\hline Lateral & $-0.100+1.25 i$ & $-1.27+18.5 i$ & $-1.095+13.69 i$ & $-2.11+26.4 i$ \\
trans. (sway) & $-0.100-1.25 i$ & $-1.27-18.5 i$ & $-1.095-13.69 i$ & $-2.11-26.4 i$ \\
\hline Lateral & -1.04 & -3.88 & -6.24 & -12.0 \\
rotational & -7.47 & -37.8 & -44.8 & -86.3
\end{tabular}

\section{Reynolds Number}

The Reynolds Number, defined as:

$$
R e=\frac{\rho v_{\text {flow }} l_{\text {chord }}}{\mu}=k_{8} \frac{\rho v_{\text {flow }} s_{l}}{\mu},
$$

where $l_{\text {chord }}$ is the aerostat chord length, is used to quantify the ratio of inertial to viscous forces in a flow and is ultimately used to classify the flow as either laminar or turbulent. While it is unlikely that water channels can be run fast enough to match Reynolds numbers observed in full-scale flight (in air, where $\left.\mu_{\text {air }}<0.02 \mu_{\text {water }}\right)$, it is also well-understood that Reynolds numbers may be off by several orders of magnitude in small-scale experiments vs. full-scale flight, while still leading to closely-matching aerodynamic behavior. The main requirement is that the flow regime at small scale matches the flow regime at full-scale (i.e., if the full-scale system is expected to exhibit a turbulent boundary layer, than so should the small scale system). Matching of boundary layer characteristic can be achieved by one of two means:

1. Ensuring that Reynolds numbers on small-scale models are in the turbulent regime, where the cutoff can be anywhere between $R e=2000$ and $R e=500,000$ (where the latter threshold corresponds to flow over a perfectly smooth flat plate).

2. Ensuring that the boundary layer on the small-scale model will be tripped from laminar to turbulent, either by incorporating a groove toward the leading edge of the model or through pre-existing properties of the model.

\section{Water Channel vs. Full-Scale System: Case Study on the Altaeros Energies Buoyant Airborne Turbine}

While the results of the last section provide a comprehensive comparison between small-scale water channel-based flight behavior and full-scale behavior, these results may seem discouraging in the sense that no two properties can be matched between the water channel and full-scale system through variation of the length scale and flow speed. For example, Table 2 shows the equivalent full-scale flow speeds at which various properties seen in the water channel are matched. The first column provides an available water channel speed, and the second, third, and fourth columns provide the flow speed at which a particular property is matched in the full-scale system.

In spite of contradictory requirements for property-matching, we have found that two key results through a case study on the Altaeros BAT:

1. Although multiple properties cannot be matched with a single choice of $v_{f l o w}, s_{l}$, and $s_{l t}$, the water channel serves as a very accurate means of predicting trends in dynamic behavior.

2. Because dynamic models can be scaled down to water channel scale and up to full-scale, the water channel results can be used to refine the scaled down dynamic model, and this model can be scaled up to full-scale to provide accurate qualitative and quantitative predictions. 
Table 2. Equivalent full-scale flow speeds at corresponding to available flow speeds in the University of Michigan $2 \mathrm{ft} \times \mathbf{2 f t}$ water channel.

\begin{tabular}{|c|c|c|c|}
\hline Water speed $(\mathrm{m} / \mathrm{s})$ & $\begin{array}{r}\text { Aero/buoyant force } \\
\text { matching speed }(\mathrm{m} / \mathrm{s})\end{array}$ & $\begin{array}{r}\text { Translational } \lambda \\
\text { matching speed }(\mathrm{m} / \mathrm{s})\end{array}$ & $\begin{array}{r}\text { Rotational } \lambda \\
\text { matching speed }(\mathrm{m} / \mathrm{s})\end{array}$ \\
\hline 0.15 & 1.73 & 35 & 20 \\
\hline 0.30 & 3.46 & 70 & 40 \\
\hline 0.45 & 5.20 & 105 & 60 \\
\hline
\end{tabular}

In order to understand the behavior of the BAT over a variety of center of mass configurations, water channel models were tested under six different ballast configurations. Table 3 provides a summary of the results of water channel testing, in comparison to simulation model predictions at water channel-scale and full-scale. Although system properties scale differently with $s_{l}$ and $v_{\text {flow }}$, one can see that the water channel is very accurate in predicting system performance.

Table 3. Summary of water channel test results and comparison with simulation results.

\begin{tabular}{rrrr} 
Ballast configuration & Water channel result & Water channel simulation & Full-scale simulation \\
\hline A & unstable & unstable & unstable \\
B & results varied & stable & stable \\
C & stable & stable & stable \\
D & stable & stable & stable \\
E & unstable & unstable & unstable \\
F & stable & stable & stable
\end{tabular}

Figs. 4-6 provide the dynamic responses for a comparison of three ballast configurations (A, C, and D), in both in the actual water channel environment (Fig. 4) and in simulation (Fig. 5 at the water channel scale and Fig. 6 at full scale). Because the water channel does not provide the ability to change flow direction, the system was perturbed laterally to evaluate its dynamic response. In simulation, the wind direction was changed, which corresponds to a different perturbation scenario but ultimately excites the same system dynamics. Several conclusions can be gleaned from the comparison of Figs. 4-6:

- The actual water channel results agreed with both water channel-scale and full-scale simulations in terms of stability and general behavior.

- The actual water channel results and water channel-scale simulation results exhibited comparable time constants, which were expectedly faster than full-scale system time constants.

- The actual water channel results differed from simulation results in terms of damping. In particular, the water channel responses exhibited more transient oscillations in some cases. Consequently, the water channel can be viewed as a good tool for predicting dynamic effects that cannot be accounted for with Altaeros' dynamic model.

\section{Conclusion}

In this paper, we have presented a novel water channel-based framework for inexpensive and accurate characterization of aerostat dynamics. This framework can be exceedingly useful for new aerostat designs that mark a significant departure from the norm and are hard to scale down to a lab scale in an air environment. Although it has been shown that important system properties scale differently as a function of fluid properties, flow speed, and scale factor, it is also shown that the water channel very accurately predicts qualitative full-scale behavior on the Altaeros BAT and, when corroborated with a dynamic model, the water channel results can be used to quantitatively predict full-scale behavior and refine the dynamic model. 


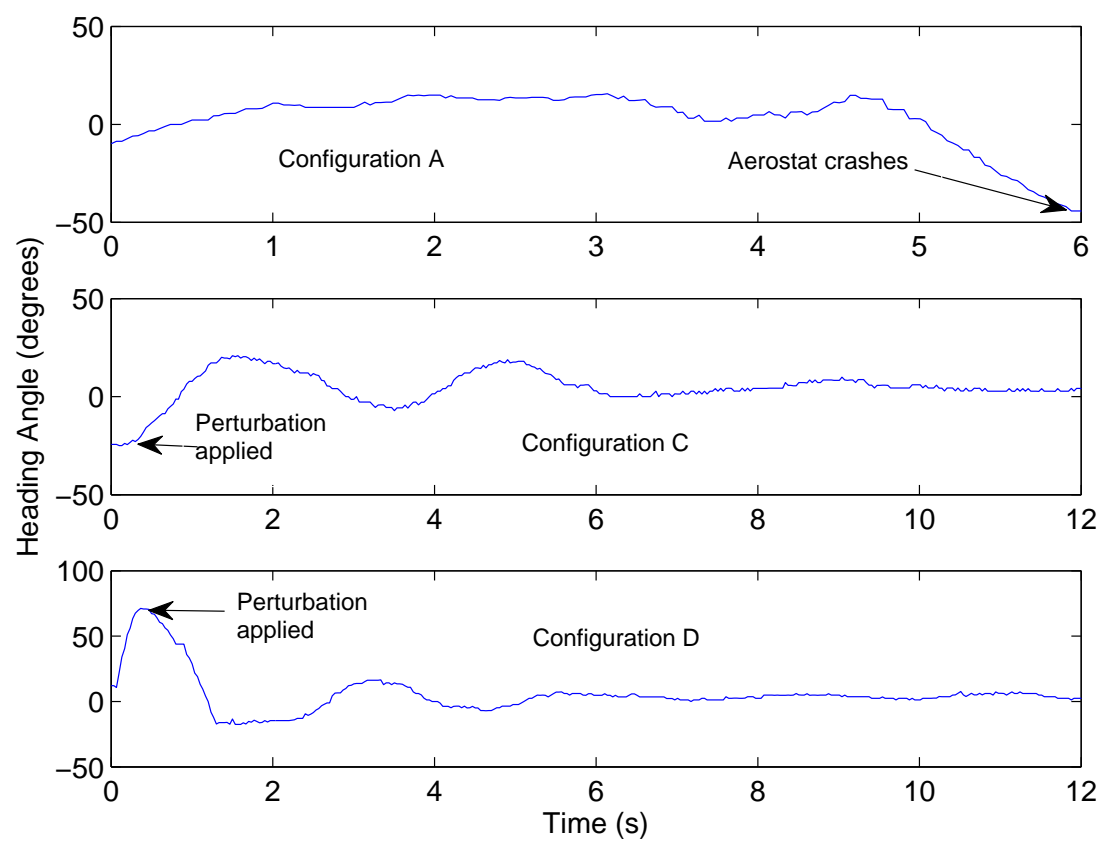

Figure 4. Dynamic response of water channel models under ballast configurations A, C, and D, at a $0.45 \frac{m}{s}$ water speed. Under ballast configuration A, the model takes an unrecoverable dive very shortly after any perturbation from the direct downwind position.
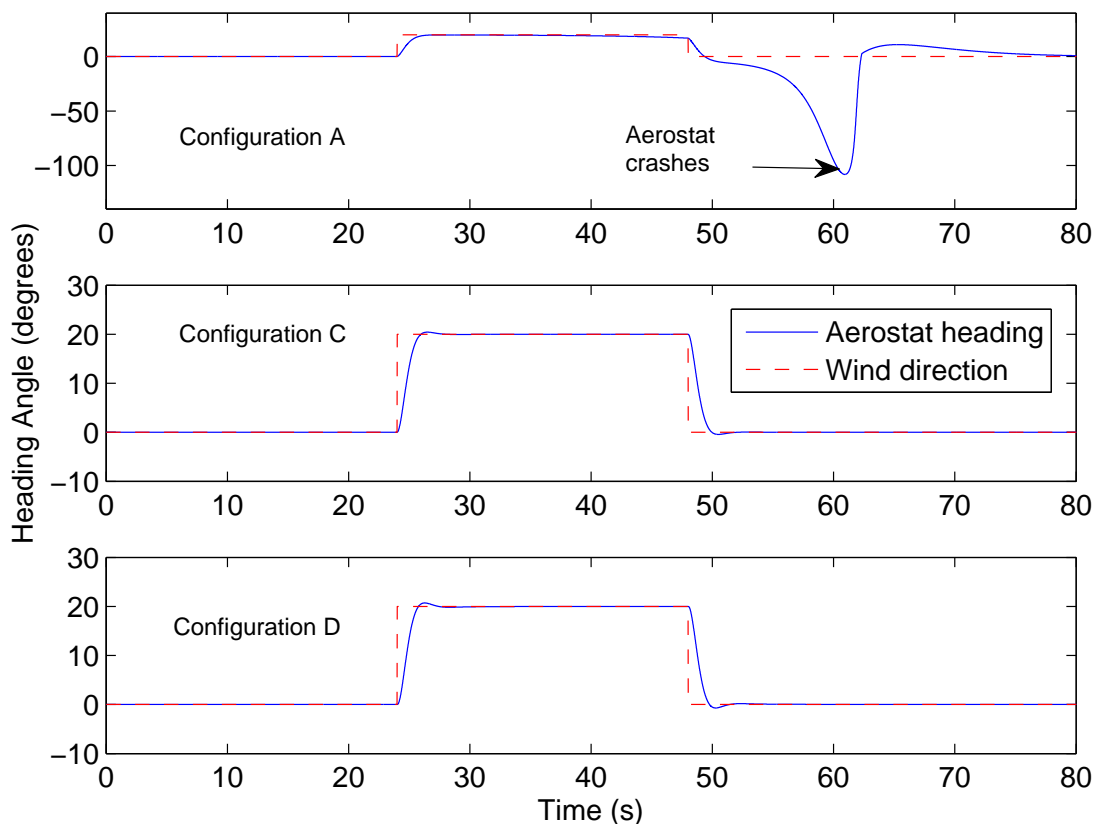

Figure 5. Simulated dynamic response of water channel models under ballast configurations A, C, and D, at a $0.45 \frac{\mathrm{m}}{\mathrm{s}}$ water speed. Under ballast configuration A, the model begins to divert from the downwind configuration on an initial flow direction change and takes an unrecoverable dive after the second direction change. After this dive, the dynamic model is no longer valid. 


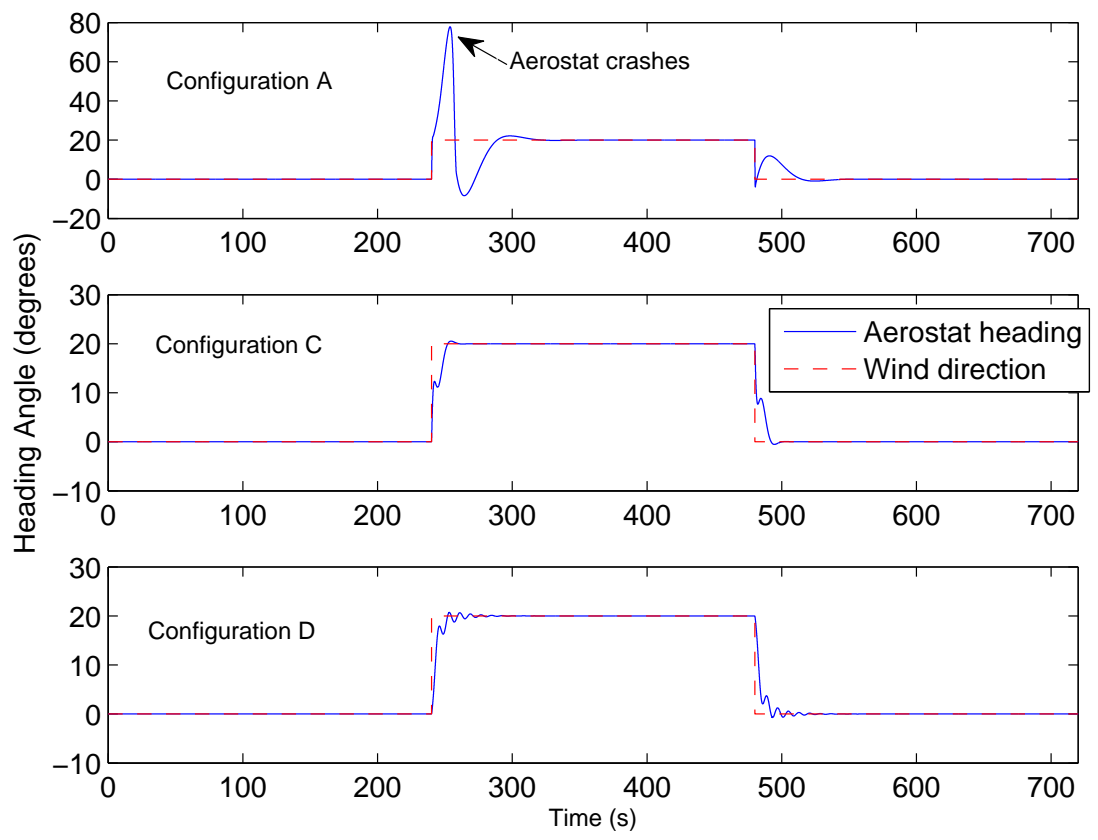

Figure 6. Simulated dynamic response of the full-scale system under ballast configurations A, C, and D, at a $10 \frac{\mathrm{m}}{\mathrm{s}}$ air speed. Under ballast configuration A, the model begins takes an unrecoverable dive after an initial wind direction change. After this dive, the dynamic model is no longer valid.

Future publications will compare both the simulation and water channel-based results with experimental flight test results to further evaluate the efficacy of the water channel framework.

\section{Acknowledgments}

The authors wish to acknowledge the entire crew at the University of Michigan who designed and built the platform for water channel testing and supported test flights, including Ian Fitzner, Brandon Bartneck, Jerry Chen, David Carter, and Prof. Pete Washabaugh. The authors also wish to thank Prof. Luis Bernal for valuable aerodynamic insight and access to the water channel and Shawn O'Grady at the University of Michigan's 3d Lab, who supported the construction of the water channel models.

\section{References}

${ }^{1}$ DeLaurier, J., "A Stability Analysis for Tethered Aerodynamically Shaped Balloons," Journal of Aircraft, Vol. 9, No. 9, 1972.

${ }^{2}$ Vorachek, J., Burbick, J. Doyle, G., "Investigation of Dynamic Behavior of Tethered Balloon Systems," Goodyear Aerospace Corporation, Technical Report No. 15497, 1972.

${ }^{3}$ Redd, T., Bland, S. Bennett, R., "Stability Analysis and Trend Study of a Balloon Tethered in a Wind, with Experimental Comparison," NASA Technical Note D-7272, 1973.

${ }^{4}$ Jones, S., Krausman, J., "Nonlinear Dynamic Simulation of a Tethered Aerostat," Journal of Aircraft, Vol. 19, No. 8, 1982, pp. 679-686.

5 Jones, S., Krausman, J., "Dynamics of the STARS Aerostat," Proceedings of the 5th AIAA Lighter-Than-Air Conference, 1983.

${ }^{6}$ Lambert, C., Nahon, M., "Stability Analysis of a Tethered Aerostat," AIAA Journal of Aircraft, Vol. 40, No. 4, pp. 705-715.

${ }^{7}$ Coulombe-Pontbriand, P., Nahon, M., "Experimental Testing and Modeling of a Tethered Spherical Aerostat in an Outdoor Environment," Journal of Wind Engineering and Industrial Aerodynamics, Vol. 97, No. 5-6, pp. 208-218.

${ }^{8}$ Miller, J., Nahon, M., "The Analysis and Design of Robust Helium Aerostats," AIAA Journal of Aircraft, Vol. 44, No. 5, pp. $1447-1458$.

${ }^{9}$ Hembree, B., Slegers, N., "Tethered Aerostat Modeling Using and Efficient Rigid-Body Dynamics Approach," Journal of Aircraft, Vol. 48, No. 2, 2011. 
${ }^{10}$ Fourie, D. A., "The Dynamic Modelling and Control System of a Tethered Aerostat for Remote Sensing Applications," Master's Thesis at the University of Stellenbosch, South Africa, 2009.

${ }^{11}$ Global Near Space Systems Website, http://www.globalnearspace.com/.

${ }^{12}$ Altaeros Energies Website, http://www.altaerosenergies.com/. 


\section{This article has been cited by:}

1. Joe Deese, Nihar Deodhar, Chris Vermillion. 2017. Nested Plant/Controller Co-Design Using G-Optimal Design and Extremum Seeking: Theoretical Framework and Application to an Airborne Wind Energy System * *This work was supported by NSF grant number 1453912, entitled CAREER: Efficient Experimental Optimization for High Performance Airborne Wind Energy Systems. IFAC-PapersOnLine 50:1, 11965-11971. [Crossref]

2. Nihar Deodhar, Alireza Bafandeh, Joe Deese, Brian Smith, Tim Muyimbwa, Christopher Vermillion, Peter Tkacik. 2017. Laboratory-Scale Flight Characterization of a Multitethered Aerostat for Wind Energy Generation. AIAA Journal 55:6, 1823-1832. [Abstract] [Full Text] [PDF] [PDF Plus]

3. Michelle Kehs, Mitchell Cobb, Hosam K. Fathy, Chris Vermillion. Insights from an experimental study on the crosswind flight of a lab-scale buoyant air turbine 5494-5499. [Crossref]

4. Zeashan Khan, Muhammad Rehan. 2016. Harnessing Airborne Wind Energy: Prospects and Challenges. Journal of Control, Automation and Electrical Systems 27:6, 728-740. [Crossref]

5. Nihar Deodhar, Chris Vermillion, Peter Tkacik. A case study in experimentally-infused plant and controller optimization for airborne wind energy systems 2371-2376. [Crossref] 